\title{
Organizing Pneumonia and Non-necrotizing Granulomata on Transbronchial Biopsy: Coexistence or Bronchiolitis Obliterans Organizing Pneumonia Secondary to Mycobacterium kansasii Disease
}

\author{
Daniel Starobin MD, Vladimir Guller MD, Alexander Gurevich MD, Gershon Fink MD, \\ Monica Huszar MD, and Sari Tal MD
}

\begin{abstract}
Mycobacterium kansasii disease was diagnosed in an 85-year-old woman admitted to the hospital for cough and gradually worsening breathlessness. Transbronchial biopsy indicated either non-necrotizing granulomata or bronchiolitis obliterans organizing pneumonia (BOOP). She was cured with combined therapy of specific anti-mycobacterial medications and systemic steroids. To our knowledge, this is the first report of $M$. kansasii non-tuberculous mycobacterium disease with a BOOPlike pattern on lung biopsy. Key words: bronchiolitis obliterans organizing pneumonia; non-tuberculous mycobacterium disease; Mycobacterium kansasii, non-necrotizing granulomata. [Respir Care 2011; 56(12):1959-1961. (C) 2011 Daedalus Enterprises]
\end{abstract}

\section{Introduction}

Bronchiolitis obliterans organizing pneumonia (BOOP), which had previously been called cryptogenic organizing pneumonia, was described in 1985 as a distinct entity. ${ }^{1,2}$ Several disorders have been described in which BOOP occurs either as a primary lesion (idiopathic BOOP) or secondary to various insults, including toxin, infection,

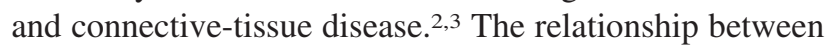
many types of infection and the onset of BOOP is still unknown. ${ }^{4}$ Non-tuberculous mycobacteria are increasingly associated with pulmonary disease. ${ }^{5}$ Mycobacterium avium intracellulare complex is rarely associated with BOOP, and we found no mention in the literature of Mycobacterium kansasii as an etiology of BOOP. ${ }^{6-8}$ To our knowl-

\footnotetext{
Drs Starobin and Fink are affiliated with the Pulmonary Institute; Drs Guller and Tal are affiliated with the Geriatrics Department; and Dr Huszar is affiliated with the Pathology Department, Kaplan Medical Center, Rehovot, Israel. Dr Gurevich is affiliated with Harzfeld Hospital, Gedera, Israel.
}

The authors have disclosed no conflicts of interest.

Correspondence: Daniel Starobin MD, Pulmonary Institute, Kaplan Medical Center, Rehovot, 76100, Israel. E-mail: daniel_s@ clalit.org.il.

DOI: $10.4187 /$ respcare.01239 edge, this is the first report of BOOP associated with $M y$ cobacterium kansasii infection.

\section{Case Report}

An 85-year-old woman with COPD, rheumatoid arthritis, hypertension, and frail performance status in the 2 weeks prior to admission presented with increasing dyspnea and cough. She had never smoked. For 10 years she had been observed by a pulmonologist for COPD and treated with inhaled corticosteroid (budesonide) and bronchodilator (formoterol). Her lung-function tests showed moderate obstructive limitation: $\mathrm{FEV}_{1} 55 \%$ of predicted and $\mathrm{FEV}_{1} /$ FVC 63\%. Previous chest radiograph and computed tomogram were both compatible with fibrotic changes of old tuberculosis. Mycobacterium kansasii had been detected in 2 of 3 sputum samples 2 years earlier, but she was not treated for that infection because there was no clinical or radiologic evidence of non-tuberculous mycobacteria disease. Bacterial susceptibility testing found the M. kansasii sensitive to rifampicine.

She had been also observed by a rheumatologist for rheumatoid arthritis, and was initially treated with methotrexate ( $7.5 \mathrm{mg}$ weekly), but had taken only 2 doses before stopping, 8 years ago, for unknown reasons. No rheumatoid arthritis activity was detected in the 6 years before admission. She had not used the immunosuppres- 


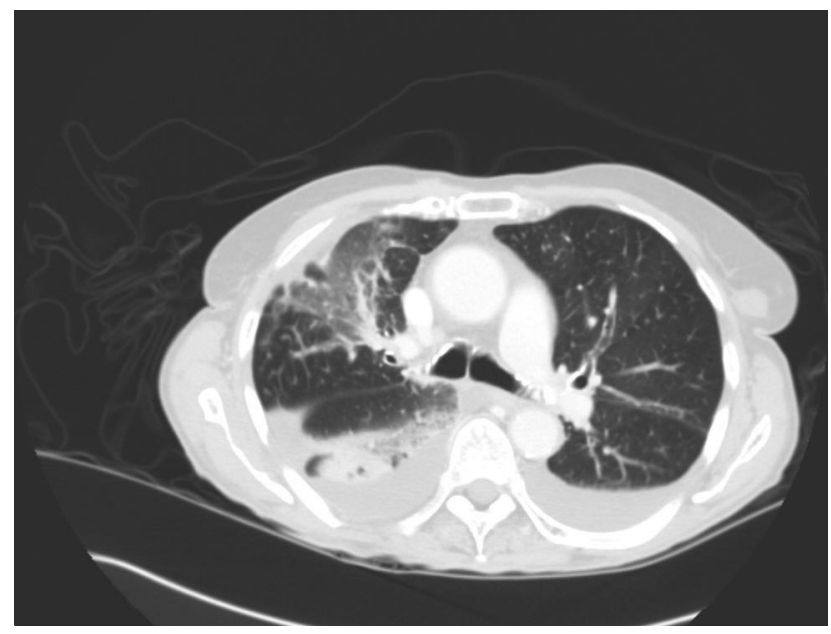

Fig. 1. Computed tomogram of the chest at the level of the carina, lung window, shows mixed ground-glass and alveolar infiltrates in the right upper and right lower lobes, and bilateral moderate-size pleural effusions.

sive medications for at least 5 years before $M$. kansasii grew in the sputum culture.

On admission she was afebrile, normotensive, in moderate respiratory distress, and $\mathrm{S}_{\mathrm{pO}_{2}}$ was $98 \%$ on $4 \mathrm{~L} / \mathrm{min}$ supplemental oxygen. Her physical examination was remarkable for dullness to percussion and decreased breath sounds over the right hemithorax. The peripheral white blood cell count was $8.4 \times 10^{3}$ cells $/ \mu \mathrm{L}$, and her hemoglobin count was $11.3 \mathrm{~g} / \mathrm{dL}$. Her erythrocyte sedimentation rate and C-reactive protein were elevated $(82 \mathrm{~mm} / \mathrm{h}$ and $29.3 \mathrm{mg} / \mathrm{dL}$, respectively). On room air her arterial blood gas values were: $\mathrm{pH} 7.45, \mathrm{P}_{\mathrm{aCO}_{2}} 50 \mathrm{~mm} \mathrm{Hg}, \mathrm{P}_{\mathrm{aO}_{2}}$ $65 \mathrm{~mm} \mathrm{Hg}$. Chest radiograph revealed pulmonary patchy alveolar infiltrates in the right upper and lower lobes and small bilateral pleural effusions.

After admission she began ceftriaxone and roxithromycin for suspected community-acquired pneumonia, and the antibiotics provided some benefit, but her respiratory symptoms showed no improvement. Sputum culture revealed mixed flora. Ziehl-Neelsen staining of 3 sputum samples and cultures of 3 sputum samples on Löwenstein-Jensen medium were negative. Blood cultures were negative. Computed tomogram of the chest revealed air-space consolidation and accompanying ground-glass attenuations in the right upper and the right lower lobes, and bilateral pleural effusions (Fig. 1). Ultrasound-guided thoracentesis retrieved exudative pleural effusion, which contained reactive mesothelial cells, macrophages, and small lymphocytes. Cultures of the pleural fluid were negative, as were the Ziehl-Neelsen staining and Löwenstein-Jensen medium tests.

On hospital day 10, because of lack of improvement, she underwent fiberoptic bronchoscopy, and the bronchoalveolar lavage fluid had ciliated cells, sheets of macro-

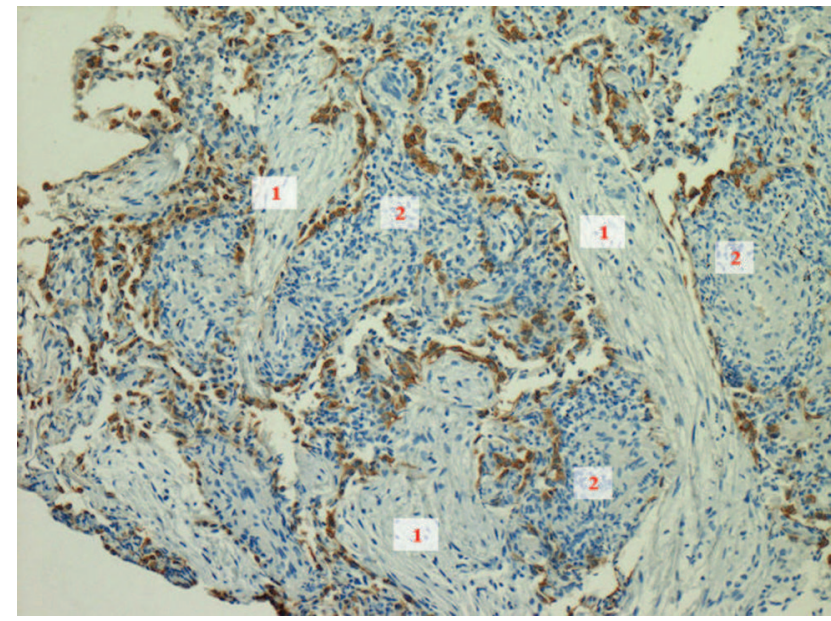

Fig. 2. Transbronchial biopsy with immunohistochemical stain for keratin (counterstain with hematoxylin, $10 \times 10$ ), shows (1) foci of organizing air-space exudate (pattern similar to bronchiolitis obliterans organizing pneumonia), and (2) indistinct non-necrotizing epitheloid granulomata.

phages, granulocytes, and lymphocytes. Transbronchial biopsy showed intra-alveolar young fibrosis tissue and granulocytic infiltrate, consistent with organizing pneumonia. The pathologist also found a few non-necrotizing granulomas in the transbronchial biopsy specimen (Fig. 2).

The empiric antibiotic therapy was stopped, and she was started on anti-tuberculosis therapy (isoniazid, ethambutol, rifampin, and pyrazinamide), which improved her respiratory symptoms. We then added prednisone (60 mg daily). Her shortness of breath ameliorated and her erythrocyte sedimentation rate and $\mathrm{C}$-reactive protein became normal. She was discharged on hospital day 30, with a recommendation to continue the isoniazid, ethambutol, and rifampin for 17 months, and to taper the prednisone dose over 9 months. Two months after hospital discharge, radiograph showed complete clearing of the pulmonary findings. She felt well, without notable cough or dyspnea.

\section{Discussion}

BOOP can be induced by various causes. ${ }^{9}$ Pulmonary and extrapulmonary causes have been described causing a "BOOP-like pattern," which is defined as plugs of fibrous tissue within small airways and alveolar ducts, typically accompanied by inflammatory changes to the bronchioles and surrounding interstitium. Some BOOP etiologies have been described in the case reports as very rare. ${ }^{10,11}$

The non-necrotizing granuloma does not exclude tuberculosis or non-tuberculous mycobacterial disease, although it is considered the hallmark of other granulomatous diseases such as sarcoidosis. ${ }^{5}$ In accordance with American Thoracic Society/Infectious Diseases Society of America 2007 guidelines, the diagnosis of non-tuberculous myco- 
bacteria disease requires clinical and radiologic signs of pulmonary disease, and bacterial evidence ( $\geq 2$ positive sputum cultures or 1 positive bronchoalveolar lavage fluid culture), and pathology evidence of mycobacterial activity.

The BOOP pattern in a transbronchial biopsy specimen was reported in a patient with Mycobacterium avium intracellulare complex. ${ }^{7}$ Coincident appearances of granulomata and organizing pneumonia in a transbronchial biopsy specimen had not been previously described in mycobacterial disease.

Our elderly female patient had few chronic illnesses and was admitted for non-specific respiratory symptoms of chronic cough and progressing dyspnea. The clinical and radiologic findings were most compatible with either lung infection or non-infectious inflammation. Her lack of response to empiric antibiotics for presumed communityacquired pneumonia led us to consider mycobacterial disease: either M. kansasii (based on previous positive sputum cultures) or tuberculosis. We chose the 4 drug anti-mycobacterial regimen after we found granulomata on transbronchial biopsy, pending the results of the bronchoalveolar lavage cultures. We discontinued pyrazinamide following negative mycobacterial cultures. Her findings did not completely fulfill the diagnostic requirements for non-tuberculous mycobacteria disease because she did not have M. kansasii growth on Löwenstein-Jensen medium during the recent disease.

The complete clinical picture of subacute disease with the radiologic and laboratory markers of active inflammation, without obvious flare-up of rheumatoid arthritis, was more probably the presentation of $M$. kansasii disease, rather than related to 2 different pulmonary illnesses appearing simultaneously. Her response to the therapy we selected convinced us that it was non-tuberculous mycobacteria disease with secondary BOOP. This case shows that BOOP pattern can be found in the transbronchial biopsy of a patient with $M$. kansasii disease. Complete cure was achieved with specific anti-mycobacterial treatment and systemic steroids, in accordance with BOOP protocol.

\section{REFERENCES}

1. Epler GR, Colby TV, McLoud TC, Carrington CB, Gaensler EA. Bronchiolitis obliterans organizing pneumonia. N Engl J Med 1985; 312(3):152-158.

2. Epler GR. Bronchiolitis obliterans organizing pneumonia. Arch Intern Med 2001;161(2):158-164.

3. Al-Ghanem S, Al-Jahdali H, Bamefleh H, Khan AN. Bronchiolitis obliterans organizing pneumonia: pathogenesis, clinical features, imaging and therapy review. Ann Thorac Med 2008;3(2):67-75.

4. Cordier JF. Organizing pneumonia. Thorax 2000;55(4):318-328.

5. Glassroth J. Pulmonary disease due to nontuberculous mycobacteria. Chest 2008;133(1):243-251.

6. Khoor A, Leslie KO, Tazelaar HD, Helmes RA, Colby TV. Diffuse pulmonary disease caused by nontuberculous mycobacteria in immunocompetent people (hot tub lung). Am J Clin Pathol 2001;115(5): $755-762$.

7. Hamada K, Nagai S, Hara Y, Hirai T, Mishima M. Pulmonary infection of Mycobacterium avium-intracellulare complex with simultaneous organizing pneumonia. Intern Med 2006;45(1):15-20.

8. Marchevsky A, Damsker B, Gribetz A, Tepper S, Geller SA. The spectrum of pathology of nontuberculous mycobacterial infections in open- lung biopsy specimens. Am J Clin Pathol 1982;78(5):695-700.

9. Cordier JF. Cryptogenic organising pneumonia. Eur Respir J 2006; 28(2):422-446.

10. Perez de Llano LA, Veres Racamonde A, Rivas Bande MJ, Piquer MO, Nieves FB, Feijoo AR. Bronchiolitis obliterans with organizing pneumonia associated with acute Coxiella burnetti infection. Respiration 2001;68(4):425-427.

11. Yale SH, Adlakha A, Sebo TJ, Ryu JH. Bronchiolitis obliterans organizing pneumonia caused by Plasmodium vivax malaria. Chest 1993;104(4):1294-1296. 